\title{
MEMS CALORIMETER FOR REAL-TIME BIOCHEMICAL TESTING AND FLUID CHARACTERIZATION
}

\author{
Yuyan Zhang and Srinivas Tadigadapa \\ Department of Electrical Engineering, Pennsylvania State University \\ University Park, PA -16802
}

\begin{abstract}
This paper presents a calorimetric microfluidic sensor which can be used to measure the molar enthalpy change of enzymatic reactions and to characterize the thermal properties of fluids at low sample volumes. The device consists of a microthermopile with the hot junctions located on a $2 \mu \mathrm{m}$ thick, freestanding silicon nitride-silicon dioxidesilicon nitride membrane and the cold junctions on bulk silicon substrate and integrated with glass microfluidic channels. The $\mathrm{p}^{+}$-polysilicon/gold microthermopiles fabricated on the thermally isolated membrane showed a sensitivity of $0.94 \mathrm{~V} / \mathrm{W}$ and a time constant of less than $100 \mathrm{~ms}$. Enzymatic catalysis of glucose, hydrogen peroxide and urea, were performed using glucose oxidase/catalase mixture, catalase, and urease respectively in the integrated microfluidic device. A sensitivity of $53.5 \mu \mathrm{V} / \mathrm{M}$ for glucose, $26.5 \mu \mathrm{V} / \mathrm{M}$ for hydrogen peroxide and $17 \mu \mathrm{V} / \mathrm{M}$ for urea was obtained. Based on the measurements a rather low detection limit for glucose of $\sim 2 \mathrm{mM}$ (30 pmole) was obtained. The primary reason for this result is the low volume of analytes sampled. The device has also been used for the measurement of the thermal conductivity and thermal diffusivity of fluids. This was achieved by introducing a periodic heat signal through the analyte and detecting the frequency dependent thermal signal response in the presence of various fluids. In this mode, the sensor design is configured to allow for the heat to primarily travel through the fluid and this coupled with the integrated temperature sensor makes a promising tool for the thermal characterization of small quantities of fluids.
\end{abstract}

\section{INTRODUCTION}

Microcalorimetry is a critical technique for the measurement of enzyme function, cell metabolism, the identification of drug effects on specific cell types, and the study of protein folding, structural transitions and other biological phenomena [1-5]. Thermopile based microcalorimeters can be configured as highly selective biochemical sensors that operate by detecting the heat of reaction to measure the concentrations of substances in gases and liquids, or the change of thermal properties such as thermal conductivity and thermal diffusivity to monitor the reaction process[2]. The device presented in this paper consists of a freestanding microthermopile integrated with glass microfluidic channels. [Figure 1] The proposed device measures the heat of reactions thermoelectrically by measuring the temperature difference created between the junctions on a freestanding membrane (hot junctions) and the junctions on the silicon substrate (cold junctions) [6]. By introducing a periodically varying heat signal in to the fluid sample using the integrated polysilicon heater, and measuring the amplitude and the phase of the heat wave traveling though the fluid due to conduction using the integrated thermopile, the thermal conductivity and the thermal diffusivity of the fluid can be accurately detected
[7]. The important criteria for obtaining high resolution here is to be able to confine the largest amount of heat flow through the test fluid. Accomplishment of this goal can be used to characterize the evolution, development, and detection of various biochemical reactions and reactants with very high sensitivity.

The thermopile is located on top of a freestanding membrane, which is fabricated from $2 \mu \mathrm{m}$ thick stress compensated dielectric layers of silicon nitride-Silicon dioxide-Silicon nitride. The thermopile itself is made from $10^{19} \mathrm{~cm}^{-3}$ p-doped polysilicon/Gold and consists of 16 thermocouples connected in series. The thermopile hot junctions are located on the freestanding membrane whereas the cold junctions of the thermopile are located on the rim of the silicon chip. The large thermal mass and good thermal conductivity of the rim keeps the cold junctions at room temperature whereas the thermally unconstrained membrane, where the reaction occurs, rises in temperature due to the heat of reaction. In addition a polysilicon heater was integrated onto the membrane. The polysilicon heater provides a technique for the calibration of the thermopile and for the measurement of the thermal properties of the fluid assays. A Pyrex 7740 glass substrate in which the $30 \mu \mathrm{m}$ deep microfluidic channels were etched was then aligned and anodically bonded onto the thermopile structure. Inlet and outlet fluidic ports were drilled in the glass substrate using a diamond drill prior to bonding. The integration of glass microfluidic channels and reaction chamber on top of the freestanding membrane allows for the introduction of controlled quantities of the analyte and enzyme, which are

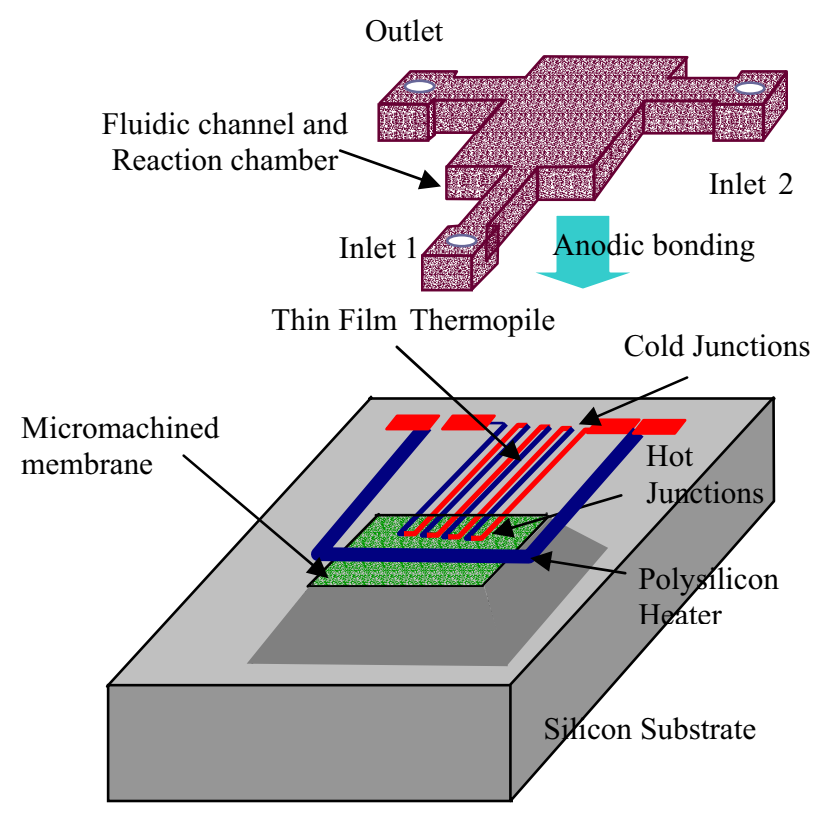

Figure 1. A three-dimensional schematic of the proposed microcalorimeter with integrated microfluidic channels.

* Travel support has been generously provided by the Transducers Research Foundation and by the DARPA MEMS and DARPA BioFlips programs 

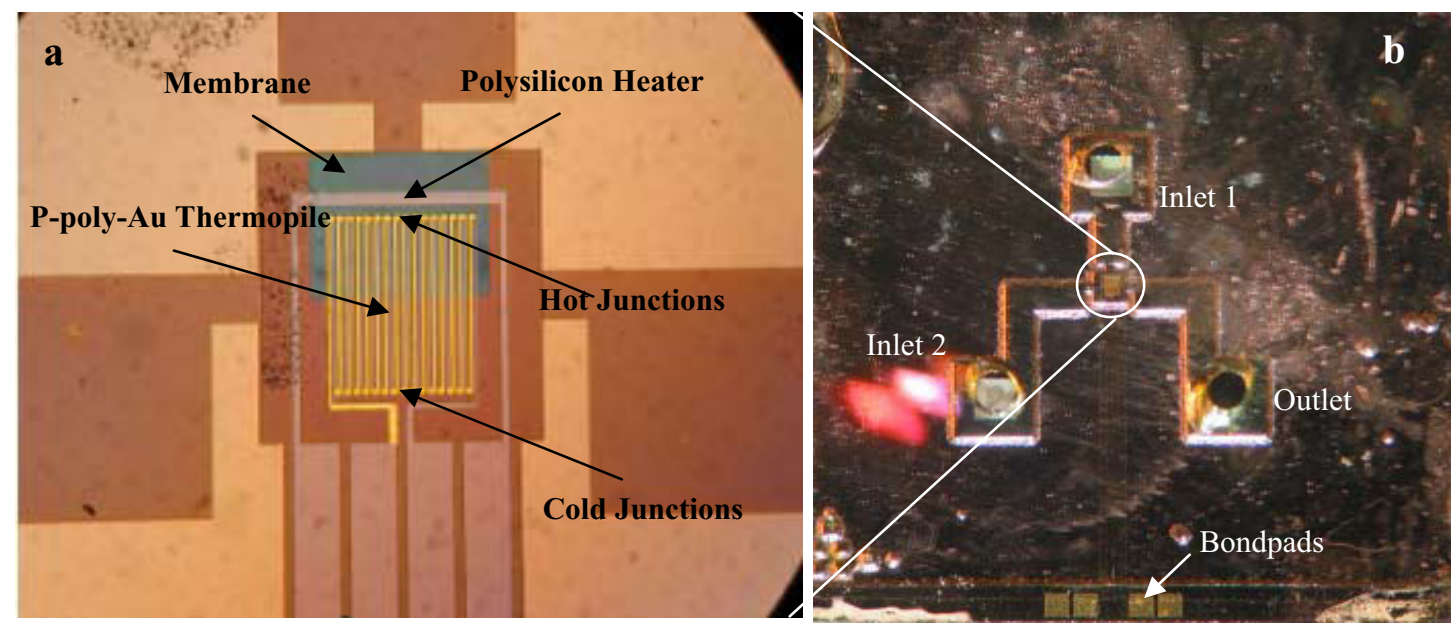

Figure 2. Optical photographs of the fabricated device. (a) Shows a close-up view of the microthermopile consisting of $16 \mathrm{Au}-\mathrm{p}^{+}$polysilicon thermocouples connected in series and the heater structure on the membrane. (b) Shows the complete microcalorimeter with the bonded glass chip and the bonding pads.

made to mix on the hot junctions by virtue of the design of the reaction chamber design. Figure 2 shows the top-vew optical pictures of the fabricated microthermopiles. A poly(dimethylsiloxane) PDMS layer is then attached on to the glass substrate that allows for easy fluidic connection to the inlet and outlet ports on the chip for testing. Using the integrated heater on the membrane, the sensitivity of the microthermopiles was measured to be $0.94 \mathrm{~V} / \mathrm{W}$ while the time constant was better than $100 \mathrm{~ms}$ [8].

\section{ENZYMATIC REACTION}

Enzymatic catalysis of glucose, hydrogen peroxide and urea, were performed using glucose oxidase-catalase, catalase, and urease respectively in continuous flow configuration using the integrated microfluidic channel. Both the substrates and enzymes were pumped into channel at a fixed rate of $0.5 \mathrm{ml} / \mathrm{hr}$, which gave out the highest output voltage for same substrate and enzyme concentration. The enthalpy changes for these three reactions are $180 \mathrm{~kJ} / \mathrm{mol}$, $100 \mathrm{~kJ} / \mathrm{mol}$ and $61 \mathrm{~kJ} / \mathrm{mol}$. Since the reaction of 1 mole of glucose to gluconic acid using glucose oxidase requires a mole of oxygen which could not be delivered in the closed glass reaction chamber, this reaction produced no output. This problem was circumvented by mixing 1moles of hydrogen peroxide with glucose solution and $800 \mathrm{U}$ of catalase enzyme with $240 \mathrm{U}$ of glucose oxidase. This resulted in the production of A sensitivity of $53.5 \mu \mathrm{V} / \mathrm{M}$ for glucose, $26.5 \mu \mathrm{V} / \mathrm{M}$ for hydrogen peroxide and $17 \mu \mathrm{V} / \mathrm{M}$ for urea was obtained. [Figure 3] Detection limit for glucose in the continuous flow mode is $\sim 2 \mathrm{mM}$ (30 pmole). This moderate sensitivity is due to the small volume of the analyte used and relatively poor mixing region in the reaction chamber, which depends on the diffusion coefficient and time. The real mixing region is two orders less than the chamber dimension.

\section{THERMAL PROPERTIES OF FLUIDS}

In addition to the direct measurement of heat of reactions, the fabricated calorimeter can also be used for the investigation of the thermal properties of the analytes. In order to accomplish this, an integrated heater is used to introduce a controlled amount of heat which travels through the freestanding membrane and the fluid on the membrane to the thermopile sensor hot junctions. The magnitude of the signal and the phase difference for periodically varying heat input can be used to determine the thermal conductivity and the thermal diffusivity of the fluid under investigation respectively.

For a system in thermal equilibrium, the temperature difference between two locations can be calculated as

$$
T\left(x_{1}\right)-T\left(x_{2}\right)=Q * R_{\text {therm }}
$$

where $Q$ is thermal flow and $R_{\text {therm }}$ is thermal resistance, which equals:

$$
R_{\text {therm }}=\frac{L}{K A}
$$

$L, A, K$ are the length, cross-sectional area and thermal conductivity of the thermal conductor respectively. A

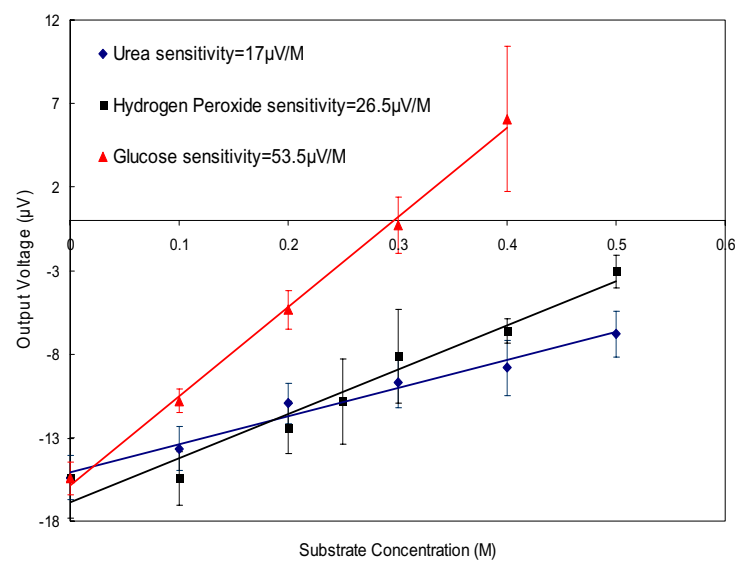

Figure 3. Output of the microthermopile as a function of urea, hydrogen peroxide, and glucose concentration catalyzed by Urease, Catalase, and Glucose Oxidase/Catalase mixture. 
simplified lumped-element model of the sensor is shown in Figure 4. Accounting for all the possible paths for heat transfer, several idealized direct thermal resistances need to be considered such as the resistance due to: (i) the air under the membrane $\left(R_{A}\right)$, (ii) the membrane $\left(R_{M}\right)$, (iii) the fluid $\left(R_{L}\right)$ from heater to thermopile. Heat also travels indirectly through fluid $\left(R_{L V}\right)$ to the glass channel $\left(R_{C}\right)$ and then back to fluid $\left(R_{L V}\right)$. Of all these four paths, the thermal resistances of fluid and membrane are the dominating ones. For the fabricated device, the membrane has an effective thermal resistance of $0.081 \mathrm{~K} / \mathrm{W}$. For the investigated fluids, the thermal resistance varied from $0.056 \mathrm{~K} / \mathrm{W}$ (DI-water) to $1.28 \mathrm{~K} / \mathrm{W}$ (air). The resolution of this technique depends upon the thermal influence the fluid has in relation to the structures constituting the sensor such as the membrane, the glass channel etc. For an accurate thermal characterization of the fluid, a significant amount of heat must flow through the fluid. The design and materials of the sensor must be chosen to maximize this possibility.

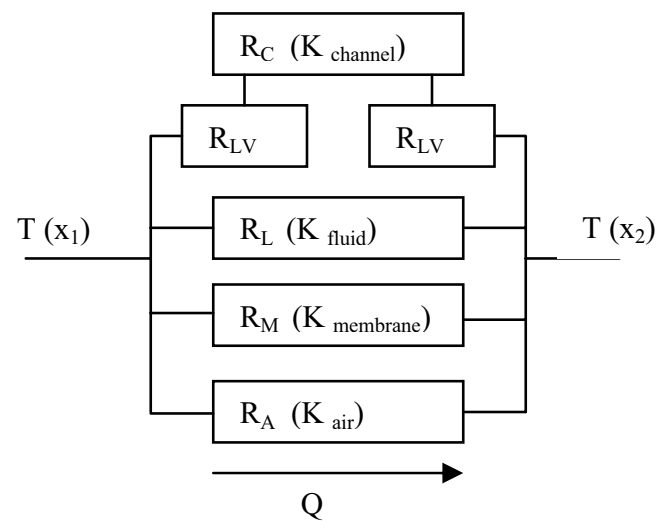

Figure 4. Lumped-element model consisting thermal resistance of channel, fluid, membrane and air.

As shown in Fig. 1 \& 2, the microthermopile structure on the membrane was integrated with a polysilicon heater. By adding a periodic voltage on the heater, a heat wave is generated on the membrane. The thermal signal detected by thermopiles is a function of the thermal properties of the liquid in the chamber. A fluid volume of around $15 \mathrm{nl}$ defined by the channel geometry was brought into contact with one side of the membrane. The measurements were carried out in quasi-stationary fluids, i.e., at a flow rate of $20 \mu \mathrm{l} / \mathrm{hr}$. This low flow rate allows for the validity of the steady state conditions and at the same time removes the influence of thermal properties change of the fluids due to the temperature increase. The heater signal voltage used was a sinusoidal function without offset. Since both the positive and the negative part of the sine wave result in heating of the polysilicon heater, the temperature function detected by the thermopile exhibits an output signal at double the input voltage frequency. An SRS830 lock-in amplifier has been used to measure the output voltage amplitude of the thermopile. The input voltage signal to the polysilicon heater of frequency $f$ was used as the reference signal and the $2 f$ signal from the microthermopile was detected. This method naturally rejects any noise at frequency $f$ by electrical coupling. Figure 5 shows the output voltage amplitude of the

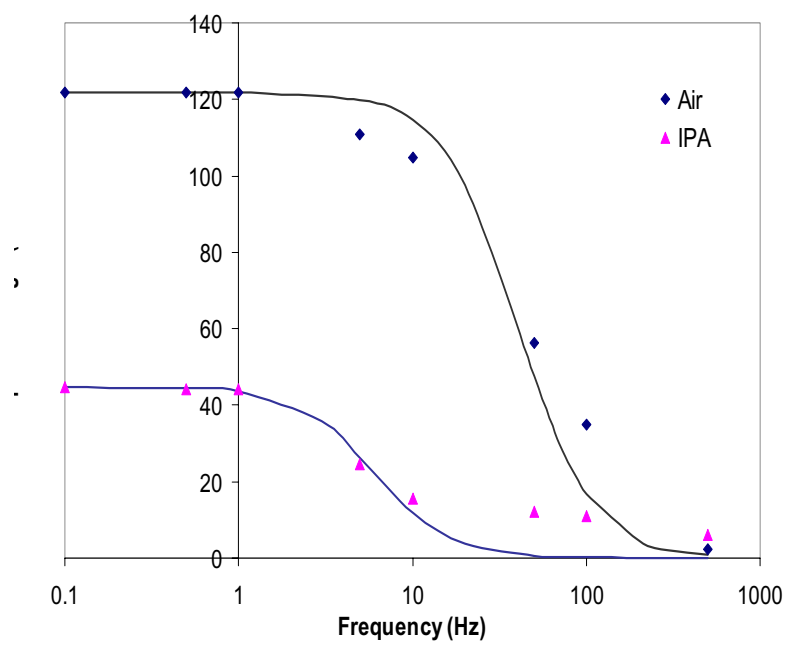

Figure 5. Thermopile output amplitude versus sinusoidal signal frequency as measured in air.

thermopile as function of the applied signal frequency in air and IPA. The dots in the figure are the measurement results and the lines represent the behavior of a first order low-pass filter. The low pass filter has a cutoff frequency of $40 \mathrm{~Hz}$ for air and $6 \mathrm{~Hz}$ in the presence of IPA. At low frequency $(<1 \mathrm{~Hz})$ the output signal does not change with frequency. Thus a time constant of $25 \mathrm{~ms}$ in air and $167 \mathrm{~ms}$ in IPA can be inferred from this measurement. As expected, the higher heat capacity of IPA results in a larger time constant.

For a fixed amplitude of the heating signal in the polysilicon heater, the output voltage of the microthermopile was measured in the presence of fluids and fluid mixtures of different thermal properties in the integrated microfluidic channel. The rms amplitude of the output signal corresponding to the effective thermal conductivity $K$ of the fluid in the microfluidic channel is shown in Figure 6. The experiments were performed at $0.1 \mathrm{~Hz}$ and $0.5 \mathrm{~Hz}$ separately with air, acetone, IPA, ethanol, water and IPA/water mixture. The two sets of results are nearly identical, which confirms

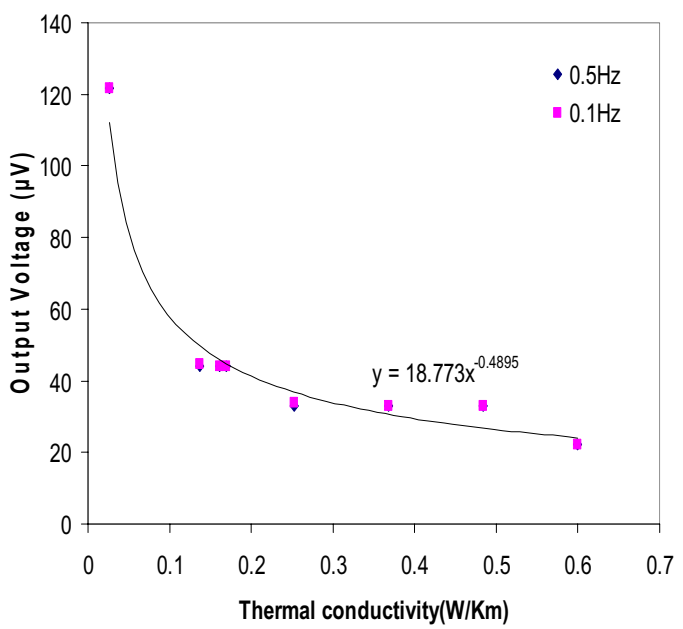

Figure 6. RMS output of the microthermopile as a function of effective thermal conductivity for air, IPA, acetone, ethanol, IPA/water, and water. 
the result from Fig, 5 for frequencies within the plateau region at low frequencies.

For a mixed fluid, the effective thermal conductivity can be calculated as [9]:

$$
K=v_{\text {water }} K_{\text {water }}+v_{I P A} K_{I P A}
$$

$v$ is the volume fraction of the two components of the mixture. All the tested fluids, and their thermal conductivities obtained from literature are shown in Table 1. The thermal conductivity and thermal response has nonlinear relationship due to the isocaloric signal approach.

\begin{tabular}{|c|c|}
\hline Fluid & $\boldsymbol{K}\left(\mathbf{W K}^{-1} \mathbf{m}^{-1}\right)$ \\
\hline Air & 0.026 \\
\hline IPA & 0.137 \\
\hline Acetone & 0.161 \\
\hline Ethanol & 0.169 \\
\hline $75 \%$ IPA+25\%water & 0.253 \\
\hline $50 \%$ IPA+50\%water & 0.369 \\
\hline 25\%IPA+75\%water & 0.484 \\
\hline DI water & 0.6 \\
\hline
\end{tabular}

Table 1. Investigated fluid and the effective thermal conductivity of the fluids and fluid mixtures investigated.

For the present design, the polysilicon heater surrounded the thermopile and thus the heat was generated all around the thermopile. Thus the generated thermal waves reaching the thermopile hot junctions are expected to have different phases. Since thermopile output signal was the average of all these heat waves, information about fluids thermal diffusivity could not be easily extracted. One of the methods of increasing the heat generated by the segment of polysilicon heater adjacent to the thermopile hot junctions is to decrease the width of this section relative to the rest of the heater structure. By this arrangement a much better phase correlation between the input and output signals was seen in a preliminary experiment. Five degrees of phase change was observed when the fluid in the microfluidic channel was changed from air to DI-water. More accurate measurements of the phase shift required for determination of the thermal diffusivity of fluids requires modifications to the design of the heater and will be reported in future studies.

\section{CONCLUSIONS}

A microthermopile calorimeter with integrated microfluidic channel has been presented, which can be used to monitor enzymatic reactions. Because of the extremely small chamber volume, the device is capable of detecting glucose solutions up to $2 \mathrm{mM}$ (30 pmole) catalyzed by glucose oxidase enzyme. The device also showed the capability of characterizing thermal properties of different fluids. In relation to other work done so far, this paper represents the smallest volumes of fluids sampled for heat of reaction and thermal property measurements. Since the total heat produced in any biochemical reaction is proportional to the volume of the reacting fluid, the sensitivity of the device is relatively low. Even using immobilized enzymes confined to the hot junction area as opposed to continuous flow configuration are not expected to improve the sensitivity of the device due to the reduced volume of the reactants. For example for a volume of $10 \mu \mathrm{l}$ and an immobilized enzyme area of $1 \mathrm{~mm}^{2}$, Herwaaden et al [6] report a sensitivity of $450 \mu \mathrm{V} / \mathrm{M}$ as opposed to the a sensitivity of $56 \mu \mathrm{V} / \mathrm{M}$ in our work using a volume of 10nl. Thus unless a temperature sensing technique capable of resolving temperatures in the sub-mK range is developed, the ultimate resolution of the thermal sensor reported here cannot exceed a $\mu \mathrm{M}$ for glucose sensing [8]. Future work for the sensor will involve improvements of the design of the sensor for more accurate measurements of the thermal diffusivity and thermal conductivity of biochemical fluids and a way to characterize biochemical reactions by real-time monitoring of the change in the thermal properties of reacting fluids.

\section{ACKNOWLEDGEMENTS}

This work is supported by a grant from the National Science Foundation, ECS-0097468.

\section{REFERENCE}

[1] Introduction to Cellular Metabolism www.mc.maricopa.edu/ tdclark/Chapter6.pdf

[2] I. Wadso, "Isothermal Microcalorimetry for the characterization of interactions between drugs and biological materials", Thermochimica Acta 267 (1995) 45-59

[3] K. Welfe, R. Misselwitz, R. Sabat, H.D. Volk, J. Schneider-Mergener, U. Reineke, and H. Welfle, "Interaction of a designed interleukin-10 epitope mimic with a antibody studied by isothermal titration microcalorimetry", J Mol Recognit 14-2(2001) 89-98

[4] B. W. Sigurskjold, "Exact analysis of competition ligand binding by displacement isothermal titration calorimetry", Anal. Biochem. 277 (2000), 260-266.

[5] P.E. Morin and E. Freire, "Direct calorimetric analysis of the enzymatic activity of yeast cytochrome c oxidase", Biochemistry 30 (1991), 8494-8500.

[6] A.W van Herwaarden, P.M. Sarro, J.W. Gardner and .Bataillard, "Liquid and Gas Micro-calorimeters for (Bio)chemical Measurements", Sensors and Actuators A, 43(1994) 24-30

[7] H. Ernst, A. Jachimowicz and G. Urban, "Dynamic Thermal Sensor-Principles in MEMS for Fluid Characterization", IEEE Sensors Journal, 1-4(2001), 361367

[8] Y. Zhang and S. Tadigadapa, "Calorimetric Biosensors with Integrated Microfluidic Channels", Biosensors and Bioelectronics, Accepted for Publication, (2004).

[9] A. Salazar, "On thermal diffusivity", Eur. J. Phys. 24 (2003), 351-358. 